Commun. Fac. Sci. Univ. Ank. Ser. A1 Math. Stat.

Volume 68, Number 2, Pages 1724-1732(2019)

DOI: $10.31801 /$ cfsuasmas.000000

ISSN 1303-5991 E-ISSN 2618-6470

http://communications.science.ankara.edu.tr/index.php?series=A1

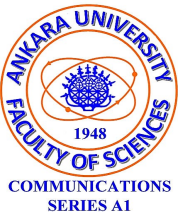

\title{
SEQUENTIALLY HAUSDORFF AND FULL SEQUENTIALLY
} HAUSDORFF SPACES

\author{
H. FULYA AKIZ AND LOKMAN KOÇAK
}

\begin{abstract}
In this paper, we define the notions of sequentially Hausdorff Space and full sequentially Hausdorff space. Also we give the relationships between these notions and Hausdorffness.
\end{abstract}

\section{INTRODUCTION}

Hausdorffness is one of the most important properties of topological spaces. A number of topological spaces encountered are Hausdorff. In a Hausdorff space, sequential limits are unique 1, 3, 4. Sequences are useful for studying functions, spaces, and other mathematical structures using the convergence properties of sequences.

On the other hand, in topology and related fields of mathematics, a sequential space is a topological space that satisfies a very feeble axiom of countability. Every open set in a topological space is also a sequentially open set [5, 6, 7. A sequential space is a space in which every sequentially open set is open. Every first countable space is a sequential space.

The notion of sequentially continuity is wider than the notion of continuity that is; every continuous function is sequential continuous. In general topology the notions of sequentially continuity, sequentially compactly and sequentially connectedness play important roles. Despite the fact that in first countable spaces these notions are equal to the notions of continuity, compactly and connectedness, respectively, there is no any generalization for other spaces. The relations between these notions give us some useful results. They are explained by using sequentially open and sequentially closed sets $8,9,10,12$. We also refer to 13,14 . The concept of G-sequential compactness was introduced in [9. On the other hand the notion of G-sequential continuity was investigated in [11] and the definition of sequential

Received by the editors: May 17, 2018; Accepted: February 13, 2019.

2010 Mathematics Subject Classification. Primary 05C38, 15A15; Secondary 05A15, 15 A18.

Key words and phrases. Hausdorff space, sequential space, sequentially open, sequentially closed.

(C)2019 Ankara University Communications Faculty of Sciences University of Ankara-Series A1 Mathematics and Statistics 
connectedness was given in [10. Also in [15], Mucuk and Şahan gave a further investigation of G-sequential continuity in topological groups.

In this study, we first define the notion of sequentially Hausdorff spaces and give some related properties and examples. We explain the relationship between Hausdorfness and sequentially Hausdorfness on topological spaces. We also define full sequentially Hausdorff spaces and give the relationships with both Hausdorff spaces and sequentially Hausdorff spaces.

\section{Preliminaries}

In a Hausdorff space, given any two distinct points, there are disjoint open sets containing the two points respectively.

Definition 2.1. 1, 2, 3, 4, Let $X=(X, \tau)$ be a topological space, $\left(x_{n}\right)$ a sequence in $X$ and $x \in X$. If, for every open neighborhood $G$ of $x$, there is a natural number $n_{0}$ such that $x_{n} \in G$ whenever $n \geq n_{0}$, then we say that the sequence $\left(x_{n}\right)$ converges to $x$.

In other words, the sequence $\left(x_{n}\right)$ converges to $x$ if $\left(x_{n}\right)$ is eventually in each open neighborhood of $x$.

Definition 2.2. 13 Let $X$ be a topological space and let $A$ be a subset of $X$. We say that $A$ is sequentially open if each sequence in $X$ converging to a point in $A$ is eventually in $A$; that is, if whenever $\left(x_{n}\right)$ is a sequence in $X$ that converges to a point in $A$, then there exists a natural number $n_{0}$ such that $x_{n}$ belongs to $A$ whenever $n \geq n_{0}$.

Definition 2.3. 13 Let $X$ be a topological space and let $F$ be a subset of $X . F$ is called sequentially closed if no sequence in $F$ converges to a point not in $F$.

By the Definition 2.2 and Definition 2.3, it is easy to see that in a topological space every open set is sequentially open and every closed set is sequentially closed.

Proposition 2.4. 13 Let $X$ be a topological space and $A \subseteq X$. Then $A$ is sequentially open if and only if $X \backslash A$ is sequentially closed.

Hence we observe the following proposition:

Proposition 2.5. [13 Let $X$ be a topological space. Then the followings are equivalent:

(i) Each sequentially open subset of $X$ is open,

(ii) Each sequentially open subset of $X$ is open,

(iii) Each subset of $X$ which intersects every convergent sequence in a closed set is closed,

(iv) Each subset of $X$ which intersects every compact metric subspace of $X$ in a closed set is closed. 
Definition 2.6. 13] Let $X$ be a topological space. Then we say $X$ is a sequential space or sequential if every sequentially open subset of $X$ is open.

Example 2.7. 13 Let $X$ be a set and $\tau$ is the discrete topology on $X$. Then the topological space $(X, \tau)$ is a sequential space.

Example 2.8. 13] Let $X$ be an uncountable set and $\tau$ is the co-countable topology on $X$. Then the topological space $(X, \tau)$ is not a sequential space.

Proposition 2.9. 13. Each subspace of a sequential space is sequential.

Definition 2.10. [5, 6, 7] Let $X$ and $Y$ be topological spaces, $f: X \rightarrow Y$ be a function and $x \in X$. If for each sequence $\left(x_{n}\right)$ converging to the point $x$, the sequence $\left(f\left(x_{n}\right)\right)$ converges to $f(x)$, then the function $f$ is called sequentially continuous.

Every continuous function is sequentially continuous.

Definition 2.11. [5, 6, 7, Let $f: X \rightarrow Y$ be a function. If for each sequentially open subset $A$ of $X, f(A)$ is sequentially open, then the function $f$ is called a sequentially open function. In a similarly way if for each sequentially closed subset $F$ of $X, f(F)$ is sequentially closed, then the function $f$ is called a sequentially closed function.

\section{Sequentially Haussdorf Spaces}

In this section, we define sequentially Haussdorff spaces and give some properties by using the notions of sequentially open set and sequentially closed set.

Definition 3.1. A topological space $X$ is called a sequentially Haussdorf space or sequentially Haussdorf if given any two distinct points $x, y$ in $X$, there exist sequentially open subsets $G \ni x$ and $H \ni y$ such that $G \cap H=\emptyset$.

Example 3.2. Since every open set is sequentially open, the discrete topology on a set is sequentially Haussdorf.

Example 3.3. Let $X \neq \emptyset$ be a set and $\tau$ be the co-countable topology on $X$. Then every subset of $X$ is sequentially open. Let $x, y \in X$ are distinct points and $G=\{x\}$. Since $y \in G^{c}$ and $G \cap G^{c}=\emptyset$, then $(X, \tau)$ is sequentially Hausdorff.

Example 3.4. Let us consider the topology $\tau=\left\{G_{a}=(a, \infty) \mid a \in \mathcal{R}\right\} \cup\{\emptyset\}$ on $\mathcal{R}$ for every $a \in \mathcal{R}$. Then the topological space $(\mathcal{R}, \tau)$ is not sequentially Hausdorff.

Example 3.5. Every first countable space is sequentially Hausdorff.

The notion of sequentially Hausdorff space is wider than the notion of Hausdorff space. We now give the following proposition:

Proposition 3.6. Every Hausdorff space is sequentially Hausdorff.

Proof: Let $(X, \tau)$ be a topological space. Since any two distinct points $x, y$ in $X$, there exist open subsets $G \ni x$ and $H \ni y$ such that $G \cap H=\emptyset$ and also every open set is sequentially open, then $(X, \tau)$ is sequentially Hausdorff. 
On the contrary, the following example shows us that every sequentially Hausdorff space does not have to be a Hausdorff space.

Example 3.7. Let $\mathcal{R}$ be the set of all real numbers and $\tau$ be the co-countable topology on $\mathcal{R}$. We know that $(X, \tau)$ is a sequentially Haussdorf space but not a Hausdorff space.

Proposition 3.8. If $(X, \tau)$ is sequentially Hausdorff, then sequential limits in $X$ are unique, that is whenever $\left(x_{n}\right)$ converges to $x$ and $y$, then $x=y$.

Proof: Suppose that $(X, \tau)$ be sequentially Hausdorff. Let $\left(x_{n}\right)$ be a sequence in $X$ converging to some point $x$ and $y$ and let $x \neq y$. Then there is a disjoint sequential open sets $G$ and $H$ such that $x \in G$ and $y \in H$. Since $G$ is sequentially open, each sequence $\left(x_{n}\right)$ in $X$ converging to $x$ is eventually in $G$; that is, there exists a natural number $n_{1}$ such that $x_{n} \in G$ whenever $n \geq n_{1}$. On the other hand since $H$ is sequentially open, each sequence $\left(x_{n}\right)$ in $X$ converging to $y$ is eventually in $H$; that is, there exists a natural number $n_{2}$ such that $x_{n} \in H$ whenever $n \geq n_{2}$. If we choose $n_{0}$ as maximum of the numbers $n_{1}$ and $n_{2}$, then there exists a natural number $n \geq n_{0}$ we obtain that $x_{n} \in G \cap H$. But we know that $G \cap H=\emptyset$. So sequential limits in $X$ are unique.

Proposition 3.9. Let $(X, \tau)$ be a sequential space. Then $(X, \tau)$ is sequentially Hausdorff if and only if $(X, \tau)$ is Hausdorff.

Proposition 3.10. Let $(X, \tau)$ be a sequentially Hausdorff space and $A \subseteq X$. Then the subspace topology $\tau_{A}$ on $A$ is sequentially Hausdorff.

Proof: The proof is clear.

Proposition 3.11. Let $(X, \tau)$ and $(Y, \sigma)$ be topological spaces and let the function $f:(X, \tau) \rightarrow(Y, \sigma)$ be sequentially open, one-to-one, onto and continuous. If $(X, \tau)$ is sequentially Hausdorff then $(Y, \sigma)$ is sequentially Hausdorff.

Proof: Let $(X, \tau)$ be sequentially Hausdorff and distinct points $y_{1}, y_{2} \in Y$. Then there exist two distinct points $x_{1}, x_{2} \in X$ such that $f\left(x_{1}\right)=y_{1}$ and $f\left(x_{2}\right)=$ $y_{2}$, since the function is one-to-one and onto. Hence, there exist sequentially open subsets $x_{1} \in G_{1}$ and $x_{2} \in G_{2}$ such that $G_{1} \cap G_{2}=\emptyset$. For the sequences $\left(x_{n}\right)$ and $\left(x_{n}^{\prime}\right)$ converging to $x_{1}$ and $x_{2}$ are eventually in $G_{1}$ and $G_{2}$, respectively. Since $f$ is continuous, so it is sequentially continuous. Then we have that the sequences $f\left(x_{n}\right)$ and $f\left(x_{n}^{\prime}\right)$ converge to $f\left(x_{1}\right)$ and $f\left(x_{2}\right)$, respectively. So they are eventually in sequential open sets $f\left(G_{1}\right)$ and $f\left(G_{2}\right)$, respectively. Since $f\left(G_{1}\right) \cap f\left(G_{2}\right)=\emptyset$, the proof is completed.

Corollary 3.12. Sequentially Hausdorffness of a space is a topological property.

Proof: Let $(X, \tau)$ and $(Y, \sigma)$ be topological spaces and the function $f:(X, \tau) \rightarrow$ $(Y, \sigma)$ be a homeomorphism; that is, $f$ be one-to-one, onto and continuous and the inverse function $f^{-1}$ be continuous. The conditions of the Proposition 3.11 are sufficient to preserve the structure of sequentially Hausdorfness. 
Definition 3.13. Let $X$ be an infinite set and $(X, \tau)$ be a topological space. Let for any two distinct points $x, y \in X$, there exist sequentially open sets $G \ni x$ and $H \ni y$ such that $G \cap H=\emptyset$. If there exist sequences $x_{n}$ and $y_{n}$, whose terms are several and converging to $x$ and $y$, respectively, such that $x_{n}$ has no term in $H$ and also $y_{n}$ has no term in $G$, then $(X, \tau)$ is called a full sequentially Hausdorff space.

Proposition 3.14. Every full sequentially Hausdorff space is sequentially Hausdorff.

Proof: By the Definition 3.13 for any two distinct points $x, y \in X$, if there are two distinct sequentially open sets $G \ni x$ and $H \ni y$ such that $G \cap H=\emptyset$, then $(X, \tau)$ is a sequentially Hausdorff space.

Example 3.15. The discrete topology on a set $X$ is full sequentially Hausdorff.

Example 3.16. Let $X \neq \emptyset$ be a set and $\tau$ be the co-countable topology on $X$. Then $(X, \tau)$ is full sequentially Hausdorff.

Example 3.17. Let us consider the usual topology $\mathcal{U}$ on $\mathcal{R}$ and distinct points $x$, $y$ and $x<y$. Let the distance between the points $x$ and $y$ be $\alpha$. Then for $x$ and $y$, there exists disjoint sequentially open sets $\left(x-\frac{\alpha}{3}, x+\frac{\alpha}{3}\right)$ and $\left(y-\frac{\alpha}{3}, y+\frac{\alpha}{3}\right)$, respectively. For a chosen sequence $\left(x_{n}\right)=\left(x-\frac{1}{n}\right)$, it has no term in $\left(y-\frac{\alpha}{3}, y+\frac{\alpha}{3}\right)$. Similarly, for a chosen sequence $\left(y_{n}\right)=\left(y+\frac{1}{n}\right)$, it has no term in $\left(x-\frac{\alpha}{3}, x+\frac{\alpha}{3}\right)$. So the usual topology on $\mathcal{R}$ is full sequentially Hausdorff.

Example 3.18. Let us consider Example 3.4. Then the topological space $(\mathcal{R}, \tau)$ is not a sequential Hausdorff space.

Proposition 3.19. Let $(X, \tau)$ be Hausdorff. If every open subset of $X$ is sequentially closed, then $(X, \tau)$ is full sequentially Hausdorff.

Proof: If $(X, \tau)$ is Hausdorff, then given any two distinct points $x, y$ in $X$, there exists sequentially open subsets $G \ni x$ and $H \ni y$ such that $G \cap H=\emptyset$. Since $G$ is sequentially closed, then there exist a sequence $\left(x_{n}\right)$ converging to $x$ is in $G$ exactly. So $H$ does not include any term of $\left(x_{n}\right)$. Since $H$ is sequentially closed, then there exists a sequence $\left(y_{n}\right)$ converging to $y$ is in $H$ exactly. So $G$ does not include any term of $\left(y_{n}\right)$.

Corollary 3.20. In a full sequentially Hausdorff space, sequential limits are unique.

Proof: We know that every full sequentially Hausdorff space is sequentially Hausdorff. By the Proposition 3.8 if $(X, \tau)$ is sequentially Hausdorff, then sequential limits in $X$ are unique.

Proposition 3.21. Let $\mathcal{R}$ be the set of real numbers and $d(x, y)=|x-y|$ be the usual metric on $\mathcal{R}$. Then $(\mathcal{R}, d)$ is full sequentially Hausdorff.

Proof: Let $x, y \in X$ and $d(x, y)=1$. Chosen open discs $G=D\left(x, \frac{1}{3}\right)$ and $H=D\left(y, \frac{1}{3}\right)$ are sequentially open neighborhoods of $x$ and $y$, respectively. It is 
easy to see that $G \cap H=\emptyset$. Then for the sequence $\left(x_{n}\right)=\left(x+\frac{2}{3 n}\right)$ converging to $x$, it has no term in $H$. Similarly for the sequence $\left(y_{n}\right)=\left(y+\frac{2}{3 n}\right)$ converging to $y$, it has no term in $G$. Then $(X, d)$ is full sequentially Hausdorff.

Proposition 3.22. Let $(X, \tau)$ and $(Y, \sigma)$ be topological spaces and let the function $f:(X, \tau) \rightarrow(Y, \sigma)$ be sequentially open, one-to-one, onto and continuous. If $(X, \tau)$ is full sequential Hausdorff then $(Y, \sigma)$ is full sequential Hausdorff.

Proof: Chosen distinct points $y_{1}, y_{2} \in Y$, since the function $f$ is one-to-one and onto, there exist two distinct points $x_{1}, x_{2} \in X$ such that $f\left(x_{1}\right)=y_{1}$ and $f\left(x_{2}\right)=y_{2}$. By the proposition 3.11 we know that $(Y, \sigma)$ is sequentially Hausdorff; that is, there exist sequentially open subsets $x_{1} \in G_{1}$ and $x_{2} \in G_{2}$ such that $G_{1} \cap$ $G_{2}=\emptyset$. Since $(X, \tau)$ is full sequentially Hausdorff, then the sequences $\left(x_{n}\right)$ and $\left(x_{n}^{\prime}\right)$ converging to $x_{1}$ and $x_{2}$ have no element in $H$ and $G$, respectively. So the sequences $\left(f\left(x_{n}\right)\right)$ and $\left(f\left(x_{n}^{\prime}\right)\right)$ converging to $f\left(x_{1}\right)$ and $f\left(x_{2}\right)$ have no element in $f(H)$ and $f(G)$, respectively, where $f(H) \ni f\left(x_{1}\right)$ and $f(G) \ni f\left(x_{2}\right)$ are sequentially open. So $(Y, \sigma)$ is also full sequentially Hausdorff.

\section{Sequentially Compact Spaces}

In this section we give a new definition of sequentially compact spaces which are defined by sequentially open sets.

Definition 4.1. Let $X$ be a topological space and $\mathcal{G}=\left\{G_{i} \mid i \in I\right\}$ be a class of any sequentially open subsets of $X$. If

$$
X \subseteq \bigcup_{i \in I} G_{i}
$$

then $\mathcal{G}$ is called a sequentially open cover of $X$.

Definition 4.2. Let $X$ be a topological space. If each of sequentially open covers of $X$ has a finite subcover, then $X$ is called a sequentially compact space.

Proposition 4.3. Every sequentially compact space is compact.

Proof: Let $X$ be a sequentially compact space and the class $\mathcal{G}=\left\{G_{i} \mid i \in I\right\}$ be an open cover of $X$. Since every open set is sequentially open, $\forall G_{i} \in \mathcal{G}$ is sequentially open. So the class $\mathcal{G}$ is a sequentially open cover of $X$. Since $X$ is sequentially compact, $\mathcal{G}$ has a finite subcover. Also it means that if $\mathcal{G}=\left\{G_{i} \mid i \in I\right\}$ be an open cover of $X$, then $\mathcal{G}$ has a a finite subcover. So $X$ is compact.

Example 4.4. Let $(X, d)$ be a metric space where $X$ is unbounded. Then $X$ is not sequentially compact. Because the class of all sequentially open sets $D(a, 1)$, for every $a \in X$, is a sequentially open cover of $X$, but does not have any finite subcover.

Example 4.5. Every finite set is sequentially compact. 
Example 4.6. Let $X$ be a nonempty set, $\tau=P(X)$ be the discrete topology on $X$ and $A$ be a subset of $X$. If $A$ is finite, then is sequentially compact. On the other hand if $A$ is infinite, then is not sequentially compact.

Example 4.7. Let $\mathcal{R}$ be the set of all real numbers and $\mathcal{U}$ be the usual topology on $\mathcal{R}$. Then $\mathcal{R}$ is not sequentially compact. Because for all natural number $n$,

$$
\mathcal{G}=\left\{G_{n} \mid n \in \mathcal{N}\right\},
$$

such that $G_{n}=(-n, n)$, is a sequentially open cover of $\mathcal{R}$. But it does not have any finite subcover.

Proposition 4.8. The finite union of sequentially compact sets in a topological space is sequentially compact.

Proof: Let $(X, \tau)$ be a topological space and $\mathcal{G}=\left\{G_{i} \mid i \in I\right\}$ be a sequentially open cover of $A=A_{1} \cup A_{2} \cup \ldots \cup A_{n}$ such that the sets $A_{1}, A_{2}, \ldots, A_{n}$ are sequentially compact. Then the class $\mathcal{G}$ is a sequentially open cover of each $A_{i}$ for $1 \leq i \leq n$. Since $A_{i}$ is sequentially compact then it has a finite cover $\mathcal{G}_{i}$. Then $\mathcal{G}^{\prime}=\mathcal{G}_{1} \cup \mathcal{G}_{2} \cup$ $\ldots \cup \mathcal{G}_{n}$ is a finite subcover of $A$. So $A$ is sequentially compact.

Proposition 4.9. Let $X$ and $Y$ be topological spaces, $f: X \rightarrow Y$ be a continuous function and $A$ be a sequentially compact subset of $X$. Then the image $f(A)$ of the set $A$ is sequentially compact in $Y$.

Proof: Let $\left\{H_{i} \mid i \in I\right\}$ be a sequentially open cover of $f(A)$. Since the function $f$ is continuous, then is sequentially continuous. So each $f^{-1}\left(H_{i}\right)$, for $i \in I$, is sequentially open. Then $\left\{f^{-1}\left(H_{i}\right) \mid i \in I\right\}$ is a sequentially open cover of $A$. Since $A$ is sequentially compact in $X$, this sequentially open cover has a finite subcover

$$
\left\{f ^ { - 1 } \left(H_{i_{1}}, f^{-1}\left(H_{i_{2}}, \ldots, f^{-1}\left(H_{i_{n}}\right\} .\right.\right.\right.
$$

Thus the finite subcover $\left\{H_{i_{1}}, H_{i_{2}}, \ldots, H_{i_{n}}\right\}$ of $\left\{H_{i} \mid i \in I\right\}$ covers $f(A)$. So $f(A)$ is sequentially compact.

Theorem 4.10. A closed subset of a sequentially compact space is sequentially compact.

Proof: Let $(X, \tau)$ be a sequentially compact space, $A$ be a closed subset of $X$ and $\mathcal{G}=\left\{G_{i} \mid i \in I\right\}$ be a sequentially open cover of $A$. Since $A$ is closed, then is sequentially closed and the class

$$
\mathcal{A}=\left\{G_{i} \mid i \in I\right\} \cup\left\{A^{c}\right\}
$$

is a sequentially open cover of $X$. Since $X$ is sequentially compact, then this sequentially open cover has a finite subcover

$$
\mathcal{A}=\left\{G_{i_{1}}, \ldots, G_{i_{n}}\right\} \cup\left\{A^{c}\right\} .
$$

So $\mathcal{G}^{\prime}=\left\{G_{i_{1}}, \ldots, G_{i_{n}}\right\}$ is a sequentially open cover of $A$ and $A$ is sequentially compact. 
Corollary 4.11. The arbitrary intersection of both closed and sequentially compact sets in a topological space is sequentially compact.

Proof: Let $X$ be a topological space and $\left\{A_{i} \mid i \in I\right\}$ be a set of compact and sequentially closed sets. For a chosen set $A_{i_{0}}$, since $A_{i_{0}}$ is sequentially compact, $\bigcap_{i \in I} A_{i}$ is closed and $\bigcap_{i \in I} A_{i} \subseteq A_{i_{0}}$, then $\bigcap_{i \in I} A_{i}$ is sequentially compact.

Theorem 4.12. A sequentially compact subset of a sequentially Hausdorff space is closed.

Proof: Let $(X, \tau)$ be a sequentially Hausdorff space and $A$ be a compact subset of $X$. We have to prove that $A^{c}$ is open. Let $x \in A^{c}$. Since $(X, \tau)$ is a sequentially Hausdorff space, then for every $a \in A$ there are sequentially open neighborhoods $G_{a}$ and $H_{a}$ of $a$ and $x$ respectively such that $G_{a} \cap H_{a}=\emptyset$. Then we obtain the class $\left\{G_{a} \mid a \in A\right\}$ and it is a sequentially open cover of $A$. Since $A$ is sequentially compact, then this sequentially open cover has a finite subcover $G_{a_{1}}, \ldots, G_{a_{n}}$; that is, $A \subseteq \bigcup_{i=1}^{n} G_{a_{i}}$. Then we have $G_{x} \cap H_{x}=\emptyset$ such that $G_{x}=\bigcup_{i=1}^{n} G_{a_{i}}$ and $H_{x}=\bigcap_{i=1}^{n} H_{a_{i}}$. Since $A \cap H_{x}=\emptyset$, then $x \in H_{x} \subseteq A^{c}$. So $A$ is closed.

Example 4.13. Let us consider the usual topology $\mathcal{U}$ on $\mathcal{R}$. Then $(\mathcal{R}, \mathcal{U})$ is sequentially Hausdorff. But the set of all rational numbers is not sequentially compact since it is not closed.

Corollary 4.14. A subset of a sequentially compact and sequentially Hausdorff space is sequentially compact if and only if it is closed.

Proof: By the Theorem 4.10 a closed subset of a sequentially compact space is sequentially compact. On the other hand by the Theorem 4.12 a sequentially compact subset of a sequentially Hausdorff space is closed.

Corollary 4.15. The arbitrary intersection of sequentially compact subsets of a sequentially Hausdorff space is sequentially compact.

Proof: Let $X$ be sequentially Hausdorff and $\left\{A_{i} \mid i \in I\right\}$ be a class of sequentially compact set. By the Theorem 4.12 each subset $A_{i}$ of $X$ is closed. Then $\bigcap_{i \in I} A_{i}$ is closed. So by the Theorem $4.10 \bigcap_{i \in I} A_{i}$ is sequentially compact.

Theorem 4.16. A function from a sequentially compact space than a sequentially Hausdorff space is closed.

Proof: Let $X$ be a sequentially compact space, $Y$ be a sequentially Hausdorff space and $f: X \rightarrow Y$ be a function. Let $K$ be closed subset of $X$. Considering the Theorem 4.10 , since $K$ is closed then is sequentially compact. Since $f$ is continuous, by the Proposition $4.9 f(K) \subseteq Y$ is sequentially compact. By the Theorem 4.12 since $Y$ is sequentially Hausdorff, then $f(K)$ is closed. So $f$ is closed.

Acknowledgments. The authors would like to thank Prof. Dr. Osman Mucuk and Dr. Tunçar Şahan for their useful comments during the preparation of this paper. 


\section{REFERENCES}

[1] Brown, R., Topology and groupoids, Booksurge PLC, 2006.

[2] Mucuk, O., Topoloji ve Kategori, Nobel Yayınları, Ankara, 2011.

[3] Rotman, J. J., An Introduction to Algebraic Topology, Springer-Verlag, New York Inc., 460 pages, 1988.

[4] Massey W., S., Algebraic Topology: An Introduction, Springer-Verlag New York Inc., 553 pages, 1990

[5] Császár, Á., $\gamma$-connected sets, Acta Mathematica Hungarica., 101, 1-2, (2003).

[6] Fedeli, A. and Le Donne, A., On good connected preimages, Topology and its Applications, 125 (2002).

[7] Huang, Q. and Lin, S., Notes on sequentially connected spaces, Acta Mathematica Hungarica, 110, 1-2 (2006).

[8] Çakallı H. and Mucuk O., On Connectedness Via A Sequential Method, Revista de la union Matematica Argentina, vol.54, (2013), 101-109.

[9] Çakallı, H., Sequential definitions of compactness, Applied Mathematics Letters., 21, 6, (2008), 594-598.

[10] Çakallı, H., Sequential definitions of connectedness, Applied Mathematics Letters., 25, (2012), 461-465.

[11] Çakallı, H., On G-continuity, Comput. Math. Appl., 61, (2011), 313-318.

[12] Huang, Q. and Lin, S., Notes on sequentially connected spaces, Acta Mathematica Hungarica, 110, 1-2, (2006), 158-164.

[13] Franklin, S. P., Spaces in which sequences suffice, Fundamenta Mathematicae, volume 57, (1965).

[14] Arhangel'skii, A. V. and Franklin, S. P. , Ordinal invariants for topological spaces, Michigan Math. J. 15 (3) (1968).

[15] Mucuk O. and Şahan T., On G-Sequential Continuity, Filomat, vol.28, (2014), 1181-1189.

Current address: H. Fulya Akız: Bozok University, Department of Mathematics, Yozgat, Turkey

E-mail address: fulya.gencel@bozok.edu.tr

ORCID Address: http://orcid.org/0000-0002-8547-2175

Current address: Lokman Koçak: Bozok University, Department of Mathematics, Yozgat, Turkey

E-mail address: $1 \mathrm{kmn} . \mathrm{kck} .40 @ \mathrm{gmail} . \mathrm{com}$

ORCID Address: http://orcid.org/0000-0002-1349-8915 